International Journal of Pure and Applied Mathematics

Volume 103 No. 1 2015, 1-17

ISSN: 1311-8080 (printed version); ISSN: 1314-3395 (on-line version)

url: http://www.ijpam.eu

doi: http://dx.doi.org/10.12732/ijpam.v103i1.1

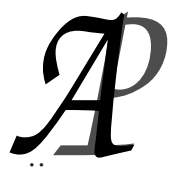

ijpam.eu

\title{
COMMON TRIPLED FIXED POINT THEOREMS UNDER WEAKER CONDITIONS
}

\author{
Bhavana Deshpande ${ }^{1 \S}$, Chetna Kothari ${ }^{2}$, Amrish Handa $^{3}$ \\ ${ }^{1,3}$ Department of Mathematics \\ Govt. P.G. Arts \& Science College \\ Ratlam (M.P.), INDIA \\ ${ }^{2}$ Department of Mathematics \\ Govt. P.G. College \\ Jaora (M.P.), INDIA
}

\begin{abstract}
We introduce the concept of (EA) property and occasionally $w$-compatibility for hybrid pair $F: X \times X \times X \rightarrow 2^{X}$ and $f: X \rightarrow X$ and establish two common tripled fixed point theorems for hybrid pair of mappings under some newly defined weaker conditions on a noncomplete metric space, which is not partially ordered. It is to be noted that to find tripled coincidence point, we do not employ the condition of continuity of any mapping involved therein. We also give an example to validate our result. We improve and generalize several known results.
\end{abstract}

AMS Subject Classification: 47H10, 54H25

Key Words: metric space, tripled fixed point, tripled coincidence point, new contractive condition, $w$-compatibility, $F$-weakly commuting mappings

Received: October 17, 2014

(C) 2015 Academic Publications, Ltd. url: www.acadpubl.eu

${ }^{\S}$ Correspondence author 


\section{Introduction and Preliminaries}

Let $(X, d)$ be a metric space and $C B(X)$ be the set of all nonempty closed bounded subsets of $X$. Let $D(x, A)$ denote the distance from $x$ to $A \subset X$ and $H$ denote the Hausdorff metric induced by $d$, that is,

$\begin{aligned} D(x, A) & =\inf _{a \in A} d(x, a), \\ \text { and } H(A, B) & =\max \left\{\sup _{a \in A} D(a, B), \sup _{b \in B} D(b, A)\right\}, \text { for all } A, B \in C B(X) .\end{aligned}$

Fixed point theorems for multivalued contractions and non-expansive mappings using the Hausdorff metric has been initiated by Markin [24] and then studied by many authors under different conditions. The theory of multivalued mappings has application in control theory, convex optimization, differential inclusions and economics.

In [11], Bhaskar and Lakshmikantham established some coupled fixed point theorems in the setting of single-valued mappings and apply these to study the existence and uniqueness of solution for periodic boundary value problems. Luong and Thuan [23] generalized the results of Bhaskar and Lakshmikantham [11]. Berinde [8] extended the results of Bhaskar and Lakshmikantham [11] and Luong and Thuan [23]. Lakshmikantham and Ciric [21] proved coupled coincidence and common coupled fixed point theorems for nonlinear contractive mappings in partially ordered complete metric spaces and extended the results of Bhaskar and Lakshmikantham [11]. Jain, Tas, Kumar and Gupta [19] extended and generalized the results of Berinde [8], Bhaskar and Lakshmikantham [11], Lakshmikantham and Ciric [21] and Luong and Thuan [23].

Recently Samet, Karapinar, Aydi and Rajic [25] claimed that most of the coupled fixed point theorems in the setting of single-valued mappings on ordered metric spaces are consequences of well-known fixed point theorems.

Berinde and Borcut [9] introduced the concept of tripled fixed point for single valued mappings in partially ordered metric spaces. In [9], Berinde and Borcut established the existence of tripled fixed point of single-valued mappings in partially ordered metric spaces.

Coupled and tripled fixed point theory for single valued mappings has a developed literature, some of these works are $[4,5,6,7,8,10,12,13,14,15$, $18,19,26]$.

The concepts related to coupled fixed point theory was introduced by Abbas, Ciric, Damjanovic and Khan [2] and obtained coupled coincidence points and common coupled fixed point theorems. 
Tripled fixed point theory for multivalued mappings was introduced by Deshpande, Sharma and Handa [16] and obtained tripled coincidence points and common tripled fixed point theorems involving hybrid pair of mappings under generalized nonlinear contraction. Very few papers were devoted to coupled and tripled fixed point problems for hybrid pair of mappings including $[2$, $16,17,22]$.

In [16], Deshpande, Sharma and Handa introduced the following for multivalued mappings:

Definition 1. Let $X$ be a nonempty set $F: X \times X \times X \rightarrow 2^{X}$ (a collection of all nonempty subsets of $X)$ and $g$ be a self-mapping on $X$. An element $(x$, $y, z) \in X \times X \times X$ is called:

(1) a tripled fixed point of $F$ if $x \in F(x, y, z), y \in F(y, z, x)$ and $z \in F(z$, $x, y)$.

(2) a tripled coincidence point of hybrid pair $\{F, g\}$ if $g(x) \in F(x, y, z)$, $g(y) \in F(y, z, x)$ and $g(z) \in F(z, x, y)$.

(3) a common tripled fixed point of hybrid pair $\{F, g\}$ if $x=g(x) \in F(x$, $y, z), y=g(y) \in F(y, z, x)$ and $z=g(z) \in F(z, x, y)$.

We denote the set of tripled coincidence points of mappings $F$ and $g$ by $C\{F, g\}$. Note that if $(x, y, z) \in C\{F, g\}$, then $(y, z, x)$ and $(z, x, y)$ are also in $C\{F, g\}$.

Definition 2. Let $F: X \times X \times X \rightarrow 2^{X}$ be a multivalued mapping and $g$ be a self-mapping on $X$. The hybrid pair $\{F, g\}$ is called $w$-compatible if $g(F(x, y, z)) \subseteq F(g x, g y, g z)$ whenever $(x, y, z) \in C\{F, g\}$.

Definition 3. Let $F: X \times X \times X \rightarrow 2^{X}$ be a multivalued mapping and $g$ be a self-mapping on $X$. The mapping $g$ is called $F$-weakly commuting at some point $(x, y, z) \in X^{3}$ if $g^{2} x \in F(g x, g y, g z), g^{2} y \in F(g y, g z, g x)$ and $g^{2} z \in F(g z, g x, g y)$.

Aamri and ElMoutawakil [1] defined a property (EA) for self-mappings which contained the class of noncompatible mappings. Kamran [20] extended the property (EA) for hybrid pair $f: X \rightarrow X$ and $T: X \rightarrow 2^{X}$. Abbas and 
Rhoades [3] extended the concept of occasionally weakly compatible mappings for hybrid pair $f: X \rightarrow X$ and $T: X \rightarrow 2^{X}$.

In this paper, we introduce the concept of (EA) property and occasionally $w$-compatibility for hybrid pair $F: X \times X \times X \rightarrow 2^{X}$ and $f: X \rightarrow X$. We establish two common tripled fixed point theorems for hybrid pair of mappings under some newly defined weaker conditions on a noncomplete metric space, which is not partially ordered. It is to be noted that to find tripled coincidence point, we do not empoly the condition of continuity of any mapping involved therein. Our results improve, extend, and generalize the results of Bhaskar and Lakshmikantham [11], Lakshmikantham and Ciric [21], Luong and Thuan [23] and many others. An example is also given to validate our results.

\section{Main Results}

We first define the following:

Definition 4. Mappings $f: X \rightarrow X$ and $F: X \times X \times X \rightarrow C B(X)$ are said to satisfy the property (EA) if there exist sequences $\left\{x_{n}\right\},\left\{y_{n}\right\},\left\{z_{n}\right\}$ in $X$, some $u, v, w$ in $X$ and $A, B, C$ in $C B(X)$ such that

$$
\begin{aligned}
\lim _{n \rightarrow \infty} f x_{n} & =u \in A=\lim _{n \rightarrow \infty} F\left(x_{n}, y_{n}, z_{n}\right), \\
\lim _{n \rightarrow \infty} f y_{n} & =v \in B=\lim _{n \rightarrow \infty} F\left(y_{n}, z_{n}, x_{n}\right), \\
\lim _{n \rightarrow \infty} f z_{n} & =w \in C=\lim _{n \rightarrow \infty} F\left(z_{n}, x_{n}, y_{n}\right) .
\end{aligned}
$$

Example 1. Let $X=[0,+\infty)$ with the usual metric. Define $g: X \rightarrow X$ and $F: X \times X \times X \rightarrow C B(X)$ by

$$
F(x, y, z)=[0,2+x+2 y+3 z] \text { and } g(x)=3 x+1, \text { for all } x, y, z \in X \text {. }
$$

Consider the sequences

$$
\left\{x_{n}\right\}=\left\{1+\frac{1}{n}\right\},\left\{y_{n}\right\}=\left\{2+\frac{1}{n}\right\},\left\{z_{n}\right\}=\left\{3+\frac{1}{n}\right\} .
$$

Clearly,

$$
\lim _{n \rightarrow \infty} g x_{n}=4 \in A=[0,16]=\lim _{n \rightarrow \infty} F\left(x_{n}, y_{n}, z_{n}\right)
$$




$$
\begin{aligned}
\lim _{n \rightarrow \infty} g y_{n} & =7 \in B=[0,13]=\lim _{n \rightarrow \infty} F\left(y_{n}, z_{n}, x_{n}\right), \\
\lim _{n \rightarrow \infty} g z_{n} & =10 \in C=[0,13]=\lim _{n \rightarrow \infty} F\left(z_{n}, x_{n}, y_{n}\right) .
\end{aligned}
$$

Therefore, $\{F, g\}$ satisfy property (EA).

Definition 5. Mappings $F: X \times X \times X \rightarrow 2^{X}$ and $f: X \rightarrow X$ are said to be occasionally $w$-compatible if and only if there exists some point $(x, y$, $z) \in X \times X \times X$ such that $f x \in F(x, y, z), f y \in F(y, z, x), f z \in F(z, x, y)$ and $f F(x, y, z) \subseteq F(f x, f y, f z)$.

Example 2. Let $X=[0,+\infty)$ with usual metric. Define $f: X \rightarrow X$, $F: X \times X \times X \rightarrow C B(X)$ by

$$
f x=\left\{\begin{aligned}
0, & 0 \leq x<1 \\
2 x, & 1 \leq x<\infty
\end{aligned}\right.
$$

and

$$
F(x, y, z)=\left\{\begin{array}{c}
{[1,1+4 x+y+z],(x, y, z) \neq(0,0,0),} \\
\{x\},(x, y, z)=(0,0,0) .
\end{array}\right.
$$

It can be easily verified that $(0,0,0)$ and $(1,1,1)$ are tripled coincidence points of $f$ and $F$, but $f F(0,0,0) \subseteq F(f 0, f 0, f 0)$ and $f F(1,1,1) \nsubseteq F(f 1, f 1, f 1)$. So the hybrid pair $\{F, f\}$ is not $w$-compatible. However, the hybrid pair $\{F$, $f\}$ is occasionally $w$-compatible.

Let $\Phi$ denote the set of all functions $\varphi:[0,+\infty) \rightarrow[0,+\infty)$ satisfying:

$\left(i_{\varphi}\right) \varphi$ is non-decreasing,

$$
\begin{aligned}
& \left(i i_{\varphi}\right) \varphi(t)<t \text { for all } t>0, \\
& \left(i i i_{\varphi}\right) \lim _{r \rightarrow t+} \varphi(r)<t \text { for all } t>0 .
\end{aligned}
$$

and $\Psi$ denote the set of all functions $\psi:[0,+\infty) \rightarrow[0,+\infty)$ which satisfies:

$$
\begin{aligned}
& \left(i_{\psi}\right) \psi \text { is continuous, } \\
& \left(i i_{\psi}\right) \psi(t)<t \text { for all } t>0 .
\end{aligned}
$$

Note that, by $\left(i_{\psi}\right)$ and $\left(i i_{\psi}\right)$ we have that $\psi(t)=0$ if and only if $t=0$.

For simplicity, we define the following:

(I) $M(x, y, z, u, v, w)$ 


$$
=\min \left\{\begin{array}{l}
D(g x, F(x, y, z)), D(g u, F(u, v, w)), \\
D(g y, F(y, z, x)), D(g v, F(v, w, u)), \\
D(g z, F(z, x, y)), D(g w, F(w, u, v)), \\
D(g x, F(u, v, w)), D(g u, F(x, y, z)), \\
D(g y, F(v, w, u)), D(g v, F(y, z, x)), \\
D(g z, F(w, u, v)), D(g w, F(z, x, y)) .
\end{array}\right\} .
$$

Theorem 1. Let $(X, d)$ be a metric space. Assume $F: X \times X \times X \rightarrow$ $C B(X)$ and $g: X \rightarrow X$ be two mappings satisfying:

(1.1) $\{F, g\}$ satisfies the property (EA) and $g(X)$ is a closed subset of $X$.

(1.2) for all $x, y, z, u, v, w \in X$, where $\varphi \in \Phi$ and $\psi \in \Psi$,

$$
\begin{aligned}
H(F(x, y, z), F(u, v, w)) \leq & \varphi[\max \{d(g x, g u), d(g y, g v), d(g z, g w)\}] \\
& +\psi[M(x, y, z, u, v, w)] .
\end{aligned}
$$

Then $F$ and $g$ have a tripled coincidence point. Moreover, if one of the following conditions holds:

(1.3) $F$ and $g$ are $w$-compatible. $\lim _{n \rightarrow \infty} g^{n} x=u, \lim _{n \rightarrow \infty} g^{n} y=v$ and $\lim _{n \rightarrow \infty} g^{n} z=w$ for some $(x, y, z) \in C\{F, g\}$ and for some $u, v, w \in X$ and $g$ is continuous at $u, v$ and $w$.

(1.4) $g$ is $F$-weakly commuting for some $(x, y, z) \in C\{F, g\}$ and $g x, g y$, $g z$ are fixed points of $g$, that is, $g^{2} x=g x, g^{2} y=g y, g^{2} z=g z$.

(1.5) $g$ is continuous at $x, y$ and $z . \lim _{n \rightarrow \infty} g^{n} u=x, \lim _{n \rightarrow \infty} g^{n} v=y$ and $\lim _{n \rightarrow \infty} g^{n} w=z$ for some $(x, y, z) \in C\{F, g\}$ and for some $u, v, w \in X$.

(1.6) $g(C\{F, g\})$ is a singleton subset of $C\{F, g\}$.

Then $F$ and $g$ have a common tripled fixed point.

Proof. Since $\{F, g\}$ satisfies the property (EA), there exist sequences $\left\{x_{n}\right\}$, $\left\{y_{n}\right\},\left\{z_{n}\right\}$ in $X$, some $u, v, w$ in $X$ and $A, B, C$ in $C B(X)$ such that

$$
\begin{aligned}
& \lim _{n \rightarrow \infty} g x_{n}=u \in A=\lim _{n \rightarrow \infty} F\left(x_{n}, y_{n}, z_{n}\right), \\
& \lim _{n \rightarrow \infty} g y_{n}=v \in B=\lim _{n \rightarrow \infty} F\left(y_{n}, z_{n}, x_{n}\right), \\
& \lim _{n \rightarrow \infty} g z_{n}=w \in C=\lim _{n \rightarrow \infty} F\left(z_{n}, x_{n}, y_{n}\right) .
\end{aligned}
$$


Since $g(X)$ is a closed subset of $X$, then there exist $x, y, z \in X$, we have

$$
u=g x, v=g y \text { and } w=g z .
$$

Now, by using condition (1.2), we get

$$
\begin{aligned}
& H\left(F\left(x_{n}, y_{n}, z_{n}\right), F(x, y, z)\right) \\
\leq & \varphi\left[\max \left\{d\left(g x_{n}, g x\right), d\left(g y_{n}, g y\right), d\left(g z_{n}, g z\right)\right\}\right] \\
+ & \psi\left[M\left(x_{n}, y_{n}, z_{n}, x, y, z\right)\right] .
\end{aligned}
$$

Letting $n \rightarrow \infty$ in the above inequality, by using (1.7), (1.8), $(I),\left(i_{\psi}\right),\left(i i_{\psi}\right)$ and $\left(i i i_{\varphi}\right)$, we get

$$
H(A, F(x, y, z)) \leq \lim _{t \rightarrow 0} \varphi(t)+0=0+0=0 .
$$

Thus

$$
H(A, F(x, y, z))=0 .
$$

Similarly, we can get

$$
H(B, F(y, z, x))=0 \text { and } H(C, F(z, x, y))=0 .
$$

Since $g x \in A, g y \in B$ and $g z \in C$ therefore

$$
g x \in F(x, y, z), g y \in F(y, z, x) \text { and } g z \in F(z, x, y)
$$

that is, $(x, y, z)$ is a tripled coincidence point of $F$ and $g$. Hence $C\{F, g\}$ is nonempty.

Suppose now that (1.3) holds. Assume that for some $(x, y, z) \in C\{F, g\}$,

$$
\lim _{n \rightarrow \infty} g^{n} x=u, \lim _{n \rightarrow \infty} g^{n} y=v \text { and } \lim _{n \rightarrow \infty} g^{n} z=w \text { where } u, v, w \in X .
$$

Since $g$ is continuous at $u, v, w$. We have, by (1.9), that $u, v$ and $w$ are fixed points of $g$, that is,

$$
g u=u, g v=v, g w=w .
$$

As $F$ and $g$ are $w$-compatible, so for all $n \geq 1$

$$
\begin{array}{ll}
g^{n} x \in & F\left(g^{n-1} x, g^{n-1} y, g^{n-1} z\right), \\
g^{n} y \in & F\left(g^{n-1} y, g^{n-1} z, g^{n-1} x\right), \\
g^{n} z \in & F\left(g^{n-1} z, g^{n-1} x, g^{n-1} y\right) .
\end{array}
$$


By using (1.2) and (1.11), we obtain

$$
\begin{aligned}
& D\left(g^{n} x, F(u, v, w)\right) \\
\leq & H\left(F\left(g^{n-1} x, g^{n-1} y, g^{n-1} z\right), F(u, v, w)\right) \\
\leq & \varphi\left[\max \left\{d\left(g^{n} x, g u\right), d\left(g^{n} y, g v\right), d\left(g^{n} z, g w\right)\right\}\right] \\
& +\psi\left[M\left(g^{n-1} x, g^{n-1} y, g^{n-1} z, u, v, w\right)\right] .
\end{aligned}
$$

On taking limit as $n \rightarrow \infty$ in the above inequality, by using (1.9), (1.10), (I), $\left(i_{\psi}\right),\left(i i_{\psi}\right)$ and $\left(i i i_{\varphi}\right)$, we get

$$
D(g u, F(u, v, w)) \leq \lim _{t \rightarrow 0} \varphi(t)+0=0+0=0,
$$

which implies that

$$
D(g u, F(u, v, w))=0 .
$$

Similarly, we can get

$$
\begin{aligned}
& D(g v, F(v, w, u))=0 \\
& D(g w, F(w, u, v))=0
\end{aligned}
$$

it follows that

$$
g u \in F(u, v, w), g v \in F(v, w, u) \text { and } g w \in F(w, u, v) .
$$

By (1.10) and (1.12), we get

$$
u=g u \in F(u, v, w), v=g v \in F(v, w, u) \text { and } w=g w \in F(w, u, v)
$$

that is, $(u, v, w)$ is a common tripled fixed point of $F$ and $g$.

Suppose now that (1.4) holds. Assume that for some $(x, y, z) \in C\{F, g\}$, $g$ is $F$-weakly commuting, that is $g^{2} x \in F(g x, g y, g z), g^{2} y \in F(g y, g z, g x)$, $g^{2} z \in F(g z, g x, g y)$ and $g^{2} x=g x, g^{2} y=g y, g^{2} z=g z$. Thus $g x=g^{2} x \in F(g x$, $g y, g z)$ and $g y=g^{2} y \in F(g y, g z, g x)$ and $g z=g^{2} z \in F(g z, g x, g y)$, that is, $(g x, g y, g z)$ is a common tripled fixed point of $F$ and $g$.

Suppose now that (1.5) holds. Assume that for some $(x, y, z) \in C\{F, g\}$ and for some $u, v, w \in X$,

$$
\lim _{n \rightarrow \infty} g^{n} u=x, \lim _{n \rightarrow \infty} g^{n} v=y \text { and } \lim _{n \rightarrow \infty} g^{n} w=z .
$$

Since $g$ is continuous at $x, y, z$. Therefore, by (1.13), $x, y$ and $z$ are fixed points of $g$, that is,

$$
g x=x, g y=y, g z=z .
$$


Since $(x, y, z) \in C\{F, g\}$. Therefore, by (1.14), we obtain

$$
x=g x \in F(x, y, z), y=g y \in F(y, z, x), z=g z \in F(z, x, y)
$$

that is, $(x, y, z)$ is a common tripled fixed point of $F$ and $g$.

Finally, suppose that (1.6) holds. Let $g(C\{F, g\})=\{(x, x, x)\}$. Then $\{x\}=\{g x\}=F(x, x, x)$. Hence $(x, x, x)$ is a common tripled fixed point of $F$ and $g$.

If we put $\psi(t)=0$ in Theorem 1 , we get the following result:

Corollary 2. Let $(X, d)$ be a metric space. Assume $F: X \times X \times X \rightarrow$ $C B(X)$ and $g: X \rightarrow X$ be two mappings satisfying (1.1) and

(2.1) for all $x, y, z, u, v, w \in X$, where $\varphi \in \Phi$,

$$
H(F(x, y, z), F(u, v, w)) \leq \varphi[\max \{d(g x, g u), d(g y, g v), d(g z, g w)\}] .
$$

Then $F$ and $g$ have a tripled coincidence point. Moreover, if one of the conditions (1.3) to (1.6) holds, then $F$ and $g$ have a common tripled fixed point.

If we put $\varphi(t)=k t$ where $0<k<1$ in Corollary 2, we get the following result:

Corollary 3. Let $(X, d)$ be a metric space. Assume $F: X \times X \times X \rightarrow$ $C B(X)$ and $g: X \rightarrow X$ be two mappings satisfying (1.1) and

(3.1) for all $x, y, z, u, v, w \in X$, where $0<k<1$,

$$
H(F(x, y, z), F(u, v, w)) \leq k \max \{d(g x, g u), d(g y, g v), d(g z, g w)\} .
$$

Then $F$ and $g$ have a tripled coincidence point. Moreover, if one of the conditions (1.3) to (1.6) holds, then $F$ and $g$ have a common tripled fixed point.

Corollary 4. Let $(X, d)$ be a metric space. Assume $F: X \times X \times X \rightarrow$ $C B(X)$ and $g: X \rightarrow X$ be two mappings satisfying (1.1) and

(4.1) for all $x, y, z, u, v, w \in X$, where $\varphi \in \Phi$ and $\psi \in \Psi$,

$$
\begin{aligned}
& H(F(x, y, z), F(u, v, w)) \\
\leq & \varphi\left[\frac{d(g x, g u)+d(g y, g v)+d(g z, g w)}{3}\right] \\
+ & \psi[M(x, y, z, u, v, w)] .
\end{aligned}
$$


Then $F$ and $g$ have a tripled coincidence point. Moreover, if one of the conditions (1.3) to (1.6) holds, then $F$ and $g$ have a common tripled fixed point.

Proof. It is clear that

$$
\begin{aligned}
& \frac{d(g x, g u)+d(g y, g v)+d(g z, g w)}{3} \\
\leq & \frac{1}{3}\left[\begin{array}{c}
\max \{d(g x, g u), d(g y, g v), d(g z, g w)\} \\
+\max \{d(g x, g u), d(g y, g v), d(g z, g w)\} \\
+\max \{d(g x, g u), d(g y, g v), d(g z, g w)\}
\end{array}\right] \\
\leq & \max \{d(g x, g u), d(g y, g v), d(g z, g w)\}
\end{aligned}
$$

Then, we apply Theorem 1 , since $\varphi$ is non-decreasing.

If we put $\psi(t)=0$ in Corollary 4 , we get the following result:

Corollary 5. Let $(X, d)$ be a metric space. Assume $F: X \times X \times X \rightarrow$ $C B(X)$ and $g: X \rightarrow X$ be two mappings satisfying (1.1) and

(5.1) for all $x, y, z, u, v, w \in X$, where $\varphi \in \Phi$,

$$
H(F(x, y, z), F(u, v, w)) \leq \varphi\left[\frac{d(g x, g u)+d(g y, g v)+d(g z, g w)}{3}\right]
$$

Then $F$ and $g$ have a tripled coincidence point. Moreover, if one of the conditions (1.3) to (1.6) holds, then $F$ and $g$ have a common tripled fixed point.

If we put $\varphi(t)=k t$ where $0<k<1$ in Corollary 5 , we get the following result:

Corollary 6. Let $(X, d)$ be a metric space. Assume $F: X \times X \times X \rightarrow$ $C B(X)$ and $g: X \rightarrow X$ be two mappings satisfying (1.1) and

(6.1) for all $x, y, z, u, v, w \in X$, where $0<k<1$,

$$
H(F(x, y, z), F(u, v, w)) \leq \frac{k}{2}[d(g x, g u)+d(g y, g v)+d(g z, g w)] .
$$

Then $F$ and $g$ have a tripled coincidence point. Moreover, if one of the conditions (1.3) to (1.6) holds, then $F$ and $g$ have a common tripled fixed point.

Theorem 7. Let $(X, d)$ be a complete metric space. Assume $F: X \times X \times$ $X \rightarrow C B(X)$ and $g: X \rightarrow X$ be mappings satisfying (1.2) and 
(7.1) $\{F, g\}$ is occasionally $w$-compatible.

Then $F$ and $g$ have a common tripled fixed point.

Proof. Since the pairs $\{F, g\}$ is occasionally $w$-compatible, therefore there exists some point $(x, y, z) \in X \times X \times X$ such that

$$
\begin{aligned}
& g x \in F(x, y, z), g y \in F(y, z, x), \\
& g z \in F(z, x, y), g F(x, y, z) \subseteq F(g x, g y, g z) .
\end{aligned}
$$

It follows that

$$
g^{2} x \in F(g x, g y, g z), g^{2} y \in F(g y, g z, g x), g^{2} z \in F(g z, g x, g y) .
$$

Now, suppose $u=g x, v=g y, w=g z$, then by (7.3), we get

$$
g u \in F(u, v, w), g v \in F(v, w, u), g w \in F(w, v, u) .
$$

Now, we shall show that $u=g u, v=g v, w=g w$. Thus, by condition (1.2) and by triangle inequality, we have

$$
\begin{aligned}
& d(g x, g u) \\
\leq & H(F(x, y, z), F(u, v, w) \\
\leq & \varphi[\max \{d(g x, g u), d(g y, g v), d(g z, g w)\}]+\psi[M(x, y, z, u, v, w)] \\
\leq & \varphi[\max \{d(g x, g u), d(g y, g v), d(g z, g w)\}] .
\end{aligned}
$$

Thus

$$
d(g x, g u) \leq \varphi[\max \{d(g x, g u), d(g y, g v), d(g z, g w)\}] .
$$

Similarly

$$
d(g y, g v) \leq \varphi[\max \{d(g x, g u), d(g y, g v), d(g z, g w)\}] .
$$

and

$$
d(g z, g w) \leq \varphi[\max \{d(g x, g u), d(g y, g v), d(g z, g w)\}] .
$$

Combining them, by $\left(i i_{\varphi}\right)$, we get

$$
\begin{aligned}
& \max \{d(g x, g u), d(g y, g v), d(g z, g w)\} \\
\leq & \varphi[\max \{d(g x, g u), d(g y, g v), d(g z, g w)\}] \\
< & \max \{d(g x, g u), d(g y, g v), d(g z, g w)\}
\end{aligned}
$$


which is a contradiction. Hence

$$
u=g x=g u, v=g y=g v, w=g z=g w .
$$

Thus, by (7.4) and (7.5), we get

$$
u=g u \in F(u, v, w), v=g v \in F(v, w, u), w=g w \in F(w, u, v),
$$

that is, $(u, v, w)$ is a common tripled fixed point of $F$ and $g$.

If we put $\psi(t)=0$ in Theorem 7, we get the following result:

Corollary 8. Let $(X, d)$ be a metric space. Assume $F: X \times X \times X \rightarrow$ $C B(X)$ and $g: X \rightarrow X$ be two mappings satisfying (2.1) and (7.1), then $F$ and $g$ have a common tripled fixed point.

If we put $\varphi(t)=k t$ where $0<k<1$ in Corollary 8, we get the following result:

Corollary 9. Let $(X, d)$ be a metric space. Assume $F: X \times X \times X \rightarrow$ $C B(X)$ and $g: X \rightarrow X$ be two mappings satisfying (3.1) and (7.1), then $F$ and $g$ have a common tripled fixed point.

Corollary 10. Let $(X, d)$ be a metric space. Assume $F: X \times X \times X \rightarrow$ $C B(X)$ and $g: X \rightarrow X$ be two mappings satisfying (4.1) and (7.1), then $F$ and $g$ have a common tripled fixed point.

Proof. It is clear that

$$
\begin{aligned}
& \frac{d(g x, g u)+d(g y, g v)+d(g z, g w)}{3} \\
\leq & \frac{1}{3}\left[\begin{array}{l}
\max \{d(g x, g u), d(g y, g v), d(g z, g w)\} \\
\max \{d(g x, g u), d(g y, g v), d(g z, g w)\} \\
\max \{d(g x, g u), d(g y, g v), d(g z, g w)\}
\end{array}\right] \\
\leq & \max \{d(g x, g u), d(g y, g v), d(g z, g w)\} .
\end{aligned}
$$

Then, we apply Theorem 7 , since $\varphi$ is non-decreasing.

If we put $\psi(t)=0$ in Corollary 10, we get the following result:

Corollary 11. Let $(X, d)$ be a metric space. Assume $F: X \times X \times X \rightarrow$ $C B(X)$ and $g: X \rightarrow X$ be two mappings satisfying (5.1) and (7.1), then $F$ and $g$ have a common tripled fixed point. result:

If we put $\varphi(t)=k t$ where $0<k<1$ in Corollary 2.11, we get the following 
Corollary 12. Let $(X, d)$ be a metric space. Assume $F: X \times X \times X \rightarrow$ $C B(X)$ and $g: X \rightarrow X$ be two mappings satisfying (6.1) and (7.1), then $F$ and $g$ have a common tripled fixed point.

Example 3. Suppose that $X=[0,1]$, equipped with the metric $d$ : $X \times X \rightarrow[0,+\infty)$ defined as $d(x, y)=\max \{x, y\}$ and $d(x, x)=0$ for all $x, y$, $z \in X$. Let $F: X \times X \times X \rightarrow C B(X)$ be defined as

$$
F(x, y, z)=\left\{\begin{array}{c}
\{0\}, \text { for } x, y, z=1 \\
{\left[0, \frac{x^{2}+y^{2}+z^{2}}{12}\right], \text { for } x, y, z \in[0,1) .}
\end{array}\right.
$$

and $g: X \rightarrow X$ be defined as

$$
g(x)=\frac{x^{2}}{2}, \text { for all } x \in X .
$$

Define $\varphi:[0,+\infty) \rightarrow[0,+\infty)$ by

$$
\varphi(t)=\left\{\begin{array}{l}
\frac{t}{2}, \text { for } t \neq 1 \\
\frac{3}{4}, \text { for } t=1
\end{array}\right.
$$

and $\psi:[0,+\infty) \rightarrow[0,+\infty)$ by

$$
\psi(t)=\frac{t}{4}, \text { for all } t \geq 0 .
$$

Now, for all $x, y, z, u, v, w \in X$ with $x, y, z, u, v, w \in[0,1)$, we have

Case $(a)$. If $x^{2}+y^{2}+z^{2}=u^{2}+v^{2}+w^{2}$, then

$$
\begin{aligned}
& H(F(x, y, z), F(u, v, w)) \\
= & \frac{u^{2}+v^{2}+w^{2}}{12} \\
\leq & \frac{1}{6} \max \left\{\frac{x^{2}}{2}, \frac{u^{2}}{2}\right\}+\frac{1}{6} \max \left\{\frac{y^{2}}{2}, \frac{v^{2}}{2}\right\}+\frac{1}{6} \max \left\{\frac{z^{2}}{2}, \frac{w^{2}}{2}\right\} \\
\leq & \frac{1}{6} d(g x, g u)+\frac{1}{6} d(g y, g v)+\frac{1}{6} d(g z, g w) \\
\leq & \frac{1}{2} \max \{d(g x, g u), d(g y, g v), d(g z, g w)\} \\
\leq & \varphi[\max \{d(g x, g u), d(g y, g v), d(g z, g w)\}] \\
& +\psi[M(x, y, z, u, v, w)] .
\end{aligned}
$$

Case (b). If $x^{2}+y^{2}+z^{2} \neq u^{2}+v^{2}+w^{2}$ with $x^{2}+y^{2}+z^{2}<u^{2}+v^{2}+w^{2}$, then

$$
H(F(x, y, z), F(u, v, w))
$$




$$
\begin{aligned}
= & \frac{u^{2}+v^{2}+w^{2}}{12} \\
\leq & \frac{1}{6} \max \left\{\frac{x^{2}}{2}, \frac{u^{2}}{2}\right\}+\frac{1}{6} \max \left\{\frac{y^{2}}{2}, \frac{v^{2}}{2}\right\}+\frac{1}{6} \max \left\{\frac{z^{2}}{2}, \frac{w^{2}}{2}\right\} \\
\leq & \frac{1}{6} d(g x, g u)+\frac{1}{6} d(g y, g v)+\frac{1}{6} d(g z, g w) \\
\leq & \frac{1}{2} \max \{d(g x, g u), d(g y, g v), d(g z, g w)\} \\
\leq & \varphi[\max \{d(g x, g u), d(g y, g v), d(g z, g w)\}] \\
& +\psi[M(x, y, z, u, v, w)] .
\end{aligned}
$$

Similarly, we obtain the same result for $u^{2}+v^{2}+w^{2}<x^{2}+y^{2}+z^{2}$. Thus the contractive condition (1.2) is satisfied for all $x, y, z, u, v, w \in X$ with $x, y, z$, $u, v, w \in[0,1)$. Again, for all $x, y, z, u, v, w \in X$ with $x, y, z \in[0,1)$ and $u$, $v, w=1$, we have

$$
\begin{aligned}
& H(F(x, y, z), F(u, v, w)) \\
= & \frac{x^{2}+y^{2}+z^{2}}{12} \\
\leq & \frac{1}{6} \max \left\{\frac{x^{2}}{2}, \frac{u^{2}}{2}\right\}+\frac{1}{6} \max \left\{\frac{y^{2}}{2}, \frac{v^{2}}{2}\right\}+\frac{1}{6} \max \left\{\frac{z^{2}}{2}, \frac{w^{2}}{2}\right\} \\
\leq & \frac{1}{6} d(g x, g u)+\frac{1}{6} d(g y, g v)+\frac{1}{6} d(g z, g w) \\
\leq & \frac{1}{2} \max \{d(g x, g u), d(g y, g v), d(g z, g w)\} \\
\leq & \varphi[\max \{d(g x, g u), d(g y, g v), d(g z, g w)\}] \\
& +\psi[M(x, y, z, u, v, w)] .
\end{aligned}
$$

Thus the contractive condition (1.2) is satisfied for all $x, y, z, u, v, w \in X$ with $x, y, z \in[0,1)$ and $u, v, w=1$. Similarly, we can see that the contractive condition (1.2) is satisfied for all $x, y, z, u, v, w \in X$ with $x, y, z, u, v, w=1$. Hence, the hybrid pair $\{F, g\}$ satisfies the contractive condition (1.2), for all $x, y, z, u, v, w \in X$. In addition, all the other conditions of Theorem 1 and Theorem 7 are satisfied and $z=(0,0,0)$ is a common tripled fixed point of hybrid pair $\{F, g\}$. The function $F: X \times X \times X \rightarrow C B(X)$ involved in this example is not continuous on $X \times X \times X$.

Remark 1. We improve, extend and generalize the result of Bhaskar and Lakshmikantham [5], Lakshmikantham and Ciric [14] and Luong and Thuan [16] in the following sense: 
(i) We prove our results in the settings of multivalued mapping and for hybrid pair of mappings.

(ii) To prove our results we consider non complete metric space and the space is also not partially ordered.

(iii) The multivalued mapping $F: X \times X \times X \rightarrow C B(X)$ is discontinuous and not satisfying mixed g-monotone property.

(iv) The function $\varphi:[0,+\infty) \rightarrow[0,+\infty)$ involved in our theorems and example is discontinuous.

$(v)$ We prove our results under some newly defined weaker conditions.

\section{References}

[1] M. Aamri and D. ElMoutawakil, Some new common fixed point theorems under strict contractive conditions. J. Math. Anal. Appl. 270 (2002), no. $1,181-188$.

[2] M. Abbas, L. Ciric, B. Damjanovic and M. A. Khan, Coupled coincidence point and common fixed point theorems for hybrid pair of mappings. Fixed Point Theory Appl. doi:10.1186/1687-1812-2012-4 (2012).

[3] . M. Abbas and B. E. Rhoades, Common Fixed Point Theorems for Hybrid Pairs of Occasionally Weakly Compatible Mappings Satisfying Generalized Contractive Condition of Integral Type. Hindawi Publishing Corporation Fixed Point Theory and Applications Volume 2007, Article ID 54101, 9 pages.

[4] S. M. Alsulami and A. Alotaibi, Tripled coincidence points for monotone operators in partially ordered metric spaces. International Mathematical Forum 7 (37) (2012), 1811-1824.

[5] H. Aydi, E. Karapinar and M. Postolache, Tripled coincidence point theorems for weak $\varphi$-contractions in partially ordered metric spaces. Fixed Point Theory and Applications 44 (2012).

[6] H. Aydi and E. Karapinar, Triple fixed points in ordered metric spaces. Bulletin of Mathematical Analysis and Applications 4 (1) (2012), 197-207.

[7] H. Aydi and E. Karapinar, New Meir-Keeler type tripled fixed point theorems on partially ordered metric spaces. Hindawi publishing corporation Mathematical Problems in Engineering Volume 2012, Article ID 409872, 17 pages. 
[8] V. Berinde, Coupled fixed point theorems for $\varphi$-contractive mixed monotone mappings in partially ordered metric spaces. Nonlinear Analysis 75 (2012), 3218-3228.

[9] V. Berinde and M. Borcut, Tripled fixed point theorems for contractive type mappings in partially ordered metric spaces. Nonlinear Anal. Theory, Methods and Applications 74 (15) (2011), 4889-4897.

[10] V. Berinde and M. Borcut, Tripled coincidence theorems of contractive type mappings in partially ordered metric spaces. Applied Mathematics and Computation 218 (10), 5929-5936.

[11] T. G. Bhaskar and V. Lakshmikantham, Fixed point theorems in partially ordered metric spaces and applications. Nonlinear Anal. 65 (7) (2006), 1379-1393.

[12] P. Charoensawan, Tripled fixed points theorems of $\varphi$-contractive mixed monotone operators on partially ordered metric spaces. Applied Mathematical Sciences 6 (105) (2012), 5229 - 5239.

[13] L. Ciric, B. Damjanovic, M. Jleli and B. Samet, Coupled fixed point theorems for generalized Mizoguchi-Takahashi contractions with applications. Fixed Point Theory and Applications 2012, 2012:51.

[14] B. Deshpande and A. Handa, Nonlinear mixed monotone-generalized contractions on partially ordered modified intuitionistic fuzzy metric spaces with application to integral equations. Afr. Mat. DOI 10.1007/s13370-0130204-0.

[15] B. Deshpande and A. Handa, Application of coupled fixed point technique in solving integral equations on modified intuitionistic fuzzy metric spaces. Hindawi Publishing Corporation Advances in Fuzzy Systems Volume 2014, Article ID 348069, 11 pages.

[16] B. Deshpande, S. Sharma and A. Handa, Tripled fixed point theorem for hybrid pair of mappings under generalized nonlinear contraction. J. Korean Soc. Math. Educ. Ser. B: Pure Appl. Math., 21 (1) (2014), 23-38.

[17] B. Deshpande and A. Handa, Generalized Mizoguchi-Takahashi contraction in consideration of common tripled fixed point theorem for hybrid pair of mappings. Accepted for publication in Malaya Journal of Matematik. 
[18] H. S. Ding, L. Li and S. Radenovic, Coupled coincidence point theorems for generalized nonlinear contraction in partially ordered metric spaces. Fixed Point Theory and Applications 2012, 2012:96.

[19] M. Jain, K. Tas, S. Kumar and N. Gupta, Coupled common fixed point results involving a $\varphi-\psi$ contractive condition for mixed g-monotone operators in partially ordered metric spaces. Journal of Inequalities and Applications 2012, 2012:285.

[20] T. Kamran, Coincidence and fixed points for hybrid strict contractions. J. Math. Anal. Appl. 299 (2004), no. 1, 235-241.

[21] V. Lakshmikantham and L. Ciric, Coupled fixed point theorems for nonlinear contractions in partially ordered metric spaces. Nonlinear Analysis: Theory, Method and Applications, 70 (12) (2009), 4341-4349.

[22] W. Long, S. Shukla and S. Radenovic, Some coupled coincidence and common fixed point results for hybrid pair of mappings in 0-complete partial metric spaces. Fixed Point Theory and Applications 2013, 2013:145.

[23] N. V. Luong and N. X. Thuan, Coupled fixed points in partially ordered metric spaces and application. Non-linear Anal. 74 (2011), 983-992.

[24] J. T. Markin, Continuous dependence of fixed point sets. Proceedings of the American Mathematical Society, 38 (1947), 545-547.

[25] B. Samet, E. Karapinar, H. Aydi and V. C. Rajic, Discussion on some coupled fixed point theorems. Fixed Point Theory and Applications 2013, 2013:50.

[26] W. Sintunavarat, P. Kumam and Y. J. Cho, Coupled fixed point theorems for nonlinear contractions without mixed monotone property. Sintunavarat et al. Fixed Point Theory and Applications 2012, 2012:170. 
